\section{IGCP Projects 2011}

(OET - on extended term)

No.507 Paleoclimates of the Cretaceous in Asia

Project leaders: Yong Il Lee (Korea), Xiaoqiao Wan (China), Takashi Sakai (Japan), Krishnan Ayyasami (India)

Duration: 2006-2010 (OET)

Website: http://igcp507.kopri.re.kr/

No. 526 Risks Resources and Record of the Past on the Continental Shelf

Project leaders: Francesco Latino Chiocci (Italy), Lindsay Collins (Australia), Michel Michaelovitch de Mahiques (Brazil), Renée Hetherington (Canada)

Duration: 2007-2011

Website: http://igcp526.io.usp.br

No.540 Gold-bearing hydrothermal fluids of oregenic deposits

Project leaders: P.S. Garofalo (Italy), J.R. Ridley (USA), Vsevolod Prokof'ev (Russia)

Duration: 2007-2011

Website: http://www.geomin.unibo.it/ igcp $\% 5$ F540/

No.546 Subduction zones of the Caribbean

Project leaders: Antonio Garcia-Casco (Spain), Uwe Martens (USA) David Buchs (Australia)

Duration: 2007-2011

Website: http://www.ugr.es/ agcasco/igcp546/

No.557 Diamonds, xenoliths and kimberlites

Project leaders: Holger Sommer (Botswana), Klaus Regenauer-Lieb (Australia), Haemyeong Jung (South Korea)

Duration: 2007 OET in 2011

Website: http://www.holgersommer.de/ IGCP 557.html

\section{No.559 Crustal Architecture and} landscape Evolution

Project leader: Bruce R. Goleby (Australia)

Duration: 2008-2012

Website: http://www.earthscrust.org

No.565 Geodetic Monitoring of the Global Water Cycle

Project leaders: Hans-Peter Plag (USA),
Richard S. Gross (USA), Markus Rothacher (Germany), Norman L. Miller (USA), Susanna Zerbini (Italy), Chris Rizos (Australia)

Duration: 2008-2012

Website: http://www.iag-ggos.org/igcp565

No. 567 Earthquake Archaeology Archaeoseismology along the AlpineHimalayan seismic zone

Project leaders: Manuel Sintubin (Belgium), Iain Stewart (United Kingdom), Tina Niemi (USA), Erhan Altunel (Turkey)

Duration: 2008-2012

Website: http://ees.kuleuven.be/igcp/567/

No. 571 Radon, health and natural hazards

Project leaders: Gavin K. Gillmore (United Kingdom), Robin G.M. Crockett (United Kingdom)

Duration: 2009-2013

Website: http://www2.northampton.ac.uk/ info/200272/radon-and-health-in-thebuilt-and-natural-environment/1008/ unescoigcp-project-571

No.572 Permian-Triassic ecosystems Project leaders: Zhong Qiang Chen (Australia), Richard J. Twitchett (United Kingdom), Jinnan Tong (China), Margaret L. Fraiser (USA), Sylvie Crasquin (France), Steve Kershaw (United Kingdom), Thomas J. Algeo (USA), Kliti Grice (Australia)

Duration: 2008-2012

Website: http://www.igcp572.org/

No. 574 Bending and Bent Orogens, and Continental Ribbons

Project leaders: Stephen T. Johnston (Canada), Gabriel Gutierrez-Alonso (Spain), Arlo Weil (USA)

Duration: 2009-2013

Website: http://www.brynmawr.edu/geology/ faculty/aweil/IGCP-574/

No. 575 Pennsylvanian terrestrial habitats and biotas of southeastern Euramerica

Project leaders: Christopher J. Cleal (United Kingdom), Stanislav Opluštil (Czech
Republic), Isabel van Waveren (The Netherlands), Mihai E. Popa (Romania), Barry A. Thomas (United Kingdom)

Duration: 2010-2014

Website: http://www.igcp575.org

No. 580 Application of magnetic susceptibility on Paleozoic sedimentary rocks

Project leaders: Anne-Christine da Silva (Belgium), Michael T. Whalen (USA), Jindrich Hladil (Czech Republic), Daizhao Chen (China), Simo Spassov (Belgium), Frederic Boulvain (Belgium), Xavier Devleeschouwer (Belgium)

Duration: 2009-2013

Website: http://www.ulg.ac.be/geolsed/MS

No. 581 Evolution of Asian River Systems Project leaders: Hongbo Zheng (China), Ryuji Tada (Japan), Peter Clift (United Kingdom), Masood Ahmad (India), Zhengxiang Li (Australia)

Duration: 2009-2013

Website: http://isg.nju.edu.cn/Exchange/Index

No. 582 Tropical Rivers: Hydro-Physical Processes, Impacts, Hazards and Management

Project leaders: Edgardo M. Latrubesse (Argentina), Rajiv Sinha (India), Jose C. Stevaux (Brazil), Zhonguan Chen (China) Duration: 2009-2013

Website: http://www.igcp582.uem.br/

No. 585 E-MARSHAL: Earth's continental MARgins: aSsessing the geoHAzard from submarine Landslides

Project leaders: Roger Urgeles (Spain), David Mosher (Canada), Jason Chaytor (USA), Michael Strasser (Germany)

Duration: 2010-2014

Website:http://www.igcp585.org

No. 586-Y Geodynamic processes in the Andes $32^{\circ}-\mathbf{3 4}{ }^{\circ} \mathrm{S}$

Project leaders: Laura Giambiagi (Argentina), Luisa Pinto (Chile), Maisa Tunik (Argentina), Sergio Sepúlveda (Chile), Stella Maris Moreiras (Argentina), Marcelo Farías (Chile), Greg Hoke (USA), Sébastien Carretier (France), Julieta Suriano (Argentina), Maximiliano Naipauer (Argentina), Victor Garcia (Argentina), Daniel Yagupsky (Argentina), Andrés Tassara (Chile)

Duration: 2010-2012 (Young Scientist Project)

Website:http://igcp586y.syr.edu/IGCP_586Y 
No.587 Entity, Facies and Time - the Ediacaran (Vendian) Puzzle

Project leaders: Patricia Vickers-Rich (Australia), Mikhail Fedonkin (Russia), Jim Gehling (Australia), Guy Narbonne (Canada)

Duration: 2010-2014

Website: http://www.geosci.monash.edu.au/ precsite

No.588 Preparing for coastal change

Project leaders: Adam D. Switzer (Malaysia), Craig Sloss (Australia), Benjamin Horton (USA), Yongqiang Zong (China)

Duration: 2010-2014

Website:http://www.coastal-change.org

No.591 The Early to Middle Palaeozoic Revolution

Project leaders: Bradley D. Cramer (USA), Pivile Pigaitë (Lithuania), Thijs R.A. Vanderbroucke (France), Kathleen Histon (Italy), Renbin Zhan (China), Guillermo L. Albanesi (Argentina), Michael J. Melchin (Canada), Mikael Calner (Sweden) Duration: 2011-2015

Website: in process

\section{No. 594 Impact of Mining on the} Environment in Africa

(Sponsored by the Swedish International Development Cooperation Agency)

Project leaders: Bohdan Kàíbek (Czech Republic), Ewa Cukrowska (South Africa), Benjamin Mapani (Namibia), Imasiku Nyambe (Zambia)

Duration: 2011-2014

Website: in process

No. 596 Climate Change and Biodiversity Patterns in the Mid-Paleozoic

Project leaders: Peter Königshof (Germany), Thomas J. Suttner (Austria), Iliana A. Boncheva (Bulgaria), Nadezhda G. Izokh (Russia), Phuong Ta Hoa (Vietnam), Thasinee Charoentitirat (Thailand), Johny
A. Waters (USA), Wolfgang Kiessling (Germany)

Duration: 2011-2015

Website: in process

No. 597 Amalgamation and Breakup Pangaa: the Type Example of the Supercontinent Cycle

Project leaders: J. Brendan Murphy (Canada), J. Duncan Keppie (Mexico), Cecilio Quesada (Spain), Bill Collins (Australia)

Duration: 2011-2015

Website: in process

No. 598 Environmental Change and Sustainability in Karst Systems

(Sponsored by the Swedish International Development Cooperation Agency)

Project leaders: Zhang Cheng (China), Augusto Auler (Brazil), Jiang Yongjun (China), Martin Knez (Slovenia), Bartolome Andreo-Navarro (Spain), Yuan Daoxian (China), Chris Groves (USA)

Duration: 2011-2014

Website: in process

\section{No.599 The Changing Early Earth}

(Sponsored by the Swedish International Development Cooperation Agency)

Project leaders: Jaana Halla (Finland), Kent C. Condie (USA), Roberto Dall'Agnol (Brazil), Mudlappa Jayananda (India), Martin J. Van Kranendonk (Australia), Hugh Rollinson (UK), Gary Stevens (South Africa), Jin-Hui Yang (China)

Duration: 2011-2014

Website: in process

\section{No. 600. Metallogenesis of Collisional Orogens}

(Sponsored by the Swedish International Development Cooperation Agency)

Project leaders: Zenqian Hou (China), David Leach (USA), Jeremy Richards (Canada), Richard Goldfarb (USA)

Duration: 2011-2014
Website: in process

No. 601 Seismotectonics and Seismic Hazards in Africa

(Sponsored by the Swedish International Development Cooperation Agency)

Project leaders: Mustapha Meghraoui (France), Vunganai Midzi (South Africa), Atalay Ayele (Ethiopia), Djillali Benouar (Algeria)

Duration: 2011-2014

Website: in process

No. 604 Groundwater and Wetlands in Ibero-America

Project leaders: Emilia Bocanegra (Argentina), Gerdon Cardoso (Brazil), Emilio Custodio (Spain), Teresita Betancur (Colombia), Marisol Manzano (Spain)

Duration: 2011-2015

Website: in process

No.606 Adressing Environmental and Health Impacts of Major and Abandoned Mines in SubSaharan Africa

(Sponsored by the Swedish International Development Cooperation Agency)

Project leaders: Theo C. Davies (South Africa), Benjamin Mapani (Namibia)

Duration: 2011-2014

Website: in process

\begin{tabular}{lr} 
Funded projects & 27 \\
O.E.T. & 2 \\
\hline Total & 29
\end{tabular}

\section{IGCP Secretariat}

UNESCO, Division of Ecological and Earth Sciences

1, Rue Miollis 75732 Paris Cedex 15 France

Tel: +33145684118

Fax: +33145685822

www.unesco.org/sciencelearth

E-mail:igcp@unesco.org 